\title{
Application of Primary Structure Method in Design of Flat Cover Cylinder Structure
}

\author{
Xinchen Wei ${ }^{a}$, Chenghong Duan ${ }^{\mathrm{b}, ~ *}$ \\ Beijing University of Chemical Technology, Beijing 100029, China \\ a553290273@qq.com, bduanchenghong@163.com
}

Keywords: design by analysis, primary structure method, finite element analysis, pressure vessel.

\begin{abstract}
The meaning of the primary structure method and its application in pressure vessel analysis design are described. In this paper, the flat cover cylinder structure is taken as the original structure. The primary structure is constructed by ANSYS software by removing the rotation constraint on the truncation plane. Compare the stress value of the original structure with the primary structure at the center of the flat cover to determine whether the stress lost by removing the rotation constraint is secondary stress. Then carry out economically design by analysis. The primary structure method has great engineering significance for the analysis and design of pressure vessel.
\end{abstract}

\section{Introduction}

Design by analysis and design by rules are two parallel pressure vessel design methods. When design by rules, the membrane stress of wall thickness is considered regardless of other types of stress. It is often uneconomical to control the local stress at a safe level by roughly using a large safety factor. Even in some cases, such as some high temperature and high pressure vessel, it's unable to control the thermal stress and mechanical stress within the level of conventional design allowable value. Design by analysis is used here to make it.

Design by analysis is a stress classification design method based on elastic stress analysis and shaping failure criterion. It is known as the "stress classification method" in recent years. The calculated results of stress are classified by location and nature, and compared with different stress limits. The design will pass when all classified stress results are less than the allowable value.

The specific approach of the stress classification is to specify the stress classification line in several dangerous areas in the structure after elastic finite element analysis. Normally the stress classification line (SCL) is perpendicular to the middle surface. Based on rule of the equivalent principle of resultant force and moment, the stress linearization method is implemented and the elastic nominal stress distributing along the SCL can be decomposed into a membrane stress, a linear bending stress and a non-linear stress. The non-linear stress is a self-equilibrated system which has no contribution to equilibrate the external loads. The disadvantage of this method is that it's impossible to distinguish the primary stress and the secondary stress by the equivalent linearization of the stress distribution curve.

In order to determine if the stress value of a structure contains secondary stress. Concept of the primary structure was firstly proposed by Lu Mingwan in literature [1], and it has been included into "The Interpretation" of JB4732-95 Steel Pressure Vessels--Design by Analysis (confirmed in 2005) [2]. It was further elaborated in articles [3, 4]. Besides, Guan Wenhua [5] applied this method in analyzing the cylindrical shell-opening flat head structure, to distinguish primary and secondary stress. In the primary structure, all the membrane stress and linear bending stress obtained from stress linearization are primary stresses.

Taking a flat cover cylinder structure as an example, this paper elaborates how to construct the primary structure based on the principle in articles $[3,4]$. If the bending moment at the edge is required to maintain the bending stress in the center region within acceptable limits, the edge bending stress is classified as $\mathrm{Pb}$; otherwise, it is classified as $\mathrm{Q}$. The three-dimensional original structure and 
primary structure are established by finite element analysis, and the structure was elaborated by using the primary structure method.

\section{Primary Structure Method}

Equivalent linearization can eliminate the nonlinear peak stress, but it needs further classification to determine which category the rest membrane stress and bending stress belong to. The primary structure method can clearly find the secondary stress. The specific approach is to find more than $1.5 \mathrm{Sm}$ maximum stress, and relief the constraints corresponding to the stress. If the new structure with removed constraint (i.e. primary structure) can still bear loads, then it is proved that the removed constraint is not necessary to help the structure to bear loads, therefore this stress due to this constraint should be classified as secondary stress.

The key of primary structure method is constructing reasonable primary structures. Primary structure can be constructed by removing some constraints. Refer to articles [3, 4], all constraints of the structures can be classified as basic constraints and redundant constraints. The basic constraints are necessary for equilibrating the mechanical loads which are not allowed to be removed. Except for basic constraints, the others are redundant constraints classified as advantageous and disadvantageous constraints. The principle of distinguishing advantageous from disadvantageous constraints is elaborated as follow. Once a redundant constraint of the structure is removed, if the primary stress in the new simplified structure becomes larger than that in the original structure, then the removed constraint is advantageous constraint, while if the maximum primary stress in the simplified structure becomes smaller than that in the original structure, then the removed constrain is disadvantageous. Advantageous redundant constraints contribute to the structure to bear loads, so retaining these advantageous constraints can result in more economical plan that costs less material.

In this paper, the flat cover cylinder structure is taken as the research object, elaborating the idea of the primary structural method, as shown in figure 1. The original structure is shown in Fig.1.a. Considering this example, there are usually two constructing methods through one cross section:

(1) Construct primary structure by releasing the continuity requirements of rotational angle and radial displacement simultaneously, referring to Fig. 1.b. By doing that, the bending moment and the transverse shearing force will disappear at the same time.

(2) Construct primary structure by just releasing the continuity requirement of rotational angle, referring to Fig.1.c. Only the bending moment disappeared, the transverse shearing force remained.

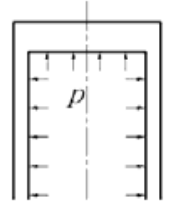

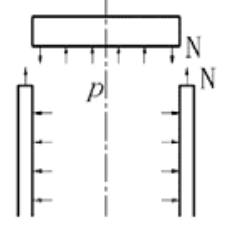

$\mathrm{b}$

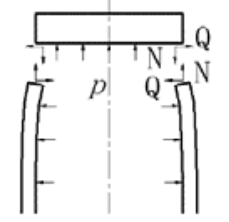

$\mathrm{c}$

Fig.1 Primary structure of flat cover cylinder structure

The primary structure shown in Fig.1.c remains only one connecting node between the cylinder shell and the flat head to transfer the internal force $\mathrm{N}$ and shearing force $\mathrm{Q}$. The originally connected interface has been cut into free surfaces. 


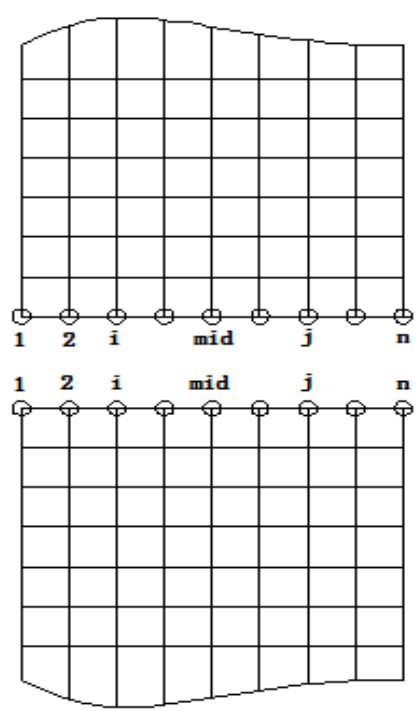

Fig. 2 Constraint equations of the truncation in the primary structure

Primary structure can be achieved by the constraint equation method in ANSYS software, and the primary structure is constructed in the form in figure 1.c in this paper. And the way of construction is shown in figure 2. Take 1/4 of flat cover cylinder structure as the model and mesh the model with solid elements. Cut the cross section off, and mesh the structure regularly to ensure that the cutting edge along the radial direction of the equidistant. Upper and lower cut section are only connected at the mid node. To avoid stress concentration caused by the single node connection, constraint equations should be applied to make the upper and lower cut section maintain flat. The constraint equations of each set of nodes along the radial direction are shown as below.

(1) The mid nodes in upper and lower cut section connect to each other, so the displacements of them are equivalent:

$$
\begin{aligned}
& u_{x \text { mid }}=u_{x \text { mid }}^{\prime} \\
& u_{y \text { mid }}=u_{y \text { mid }}^{\prime}
\end{aligned}
$$

(2) All nodes in each cut section maintain in a flat plane.

The displacement constraint equations of each node in upper cut section are:

$$
\begin{aligned}
& u_{\mathrm{x} i}=\frac{u_{\mathrm{x} i+1}+u_{x i-1}}{2} \\
& u_{\mathrm{y} i}=\frac{u_{\mathrm{y} i+1}+u_{y i-1}}{2}
\end{aligned}
$$

The displacement constraint equations of each node in lower cut section are:

$$
\begin{aligned}
& u_{\mathrm{x} i}^{\prime}=\frac{u_{\mathrm{x} i+1}^{\prime}+u_{x i-1}^{\prime}}{2} \\
& u_{\mathrm{y} i}^{\prime}=\frac{u_{\mathrm{y} i+1}^{\prime}+u_{y i-1}^{\prime}}{2}
\end{aligned}
$$

For above equations, ux and uy are radial displacement and axial displacement of each node in upper cut section. And ux' and uy' are radial displacement and axial displacement of each node in lower cut section. mid is the number of the mid node in cut sections. According to the above equations, use the command CE provided by ANSYS to establish constraint equations, and the relationship in equations (1)-(3) can be accurately expressed in the FEM modeling. So the primary structure (shown in Fig.3) can be constructed. 


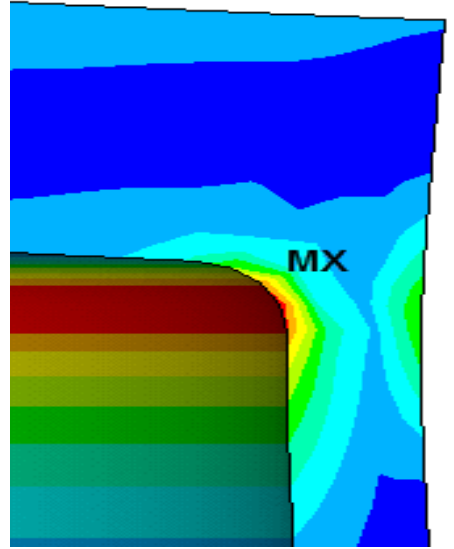

Fig. 3.a. Original structure

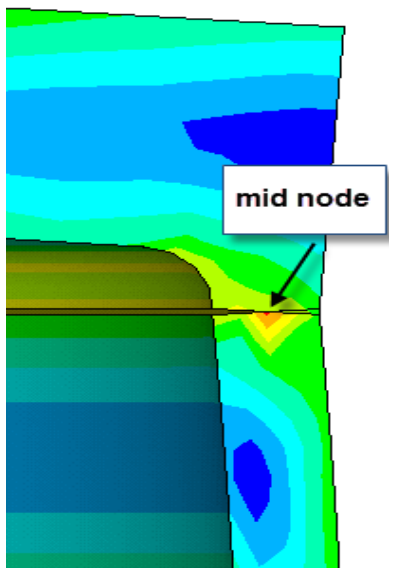

Fig. 3.b. Primary structure

Fig. 3 Construction of the primary structure

\section{Calculation and Conclusion}

\subsection{Calculation of Flat Cover Cylinder Structures}

According to JB4732-1995 [2] paragraph 9.2.2, in order to investigate the primary structure of the flat cover cylinder structure, figure out a series of seizes of the type (b) flat head based on the curve in figure 9-3. These sizes are listed in table 1. Calculated pressure $\mathrm{Pc}$ is $5.0 \mathrm{MPa}$. Flat head material is $16 \mathrm{Mn}$ forging. Cylinder material is Q345R. Take the smaller allowable stress of the former two materials as $\mathrm{Sm}$, i.e. $\mathrm{Sm}=174 \mathrm{MPa}$. The elastic modulus $\mathrm{E}=200100 \mathrm{MPa}$. The allowable value of $\mathrm{S}_{\text {II }}$ and $\mathrm{S}_{\mathrm{III}}$ is $1.5 \mathrm{Sm}=261 \mathrm{MPa}$.

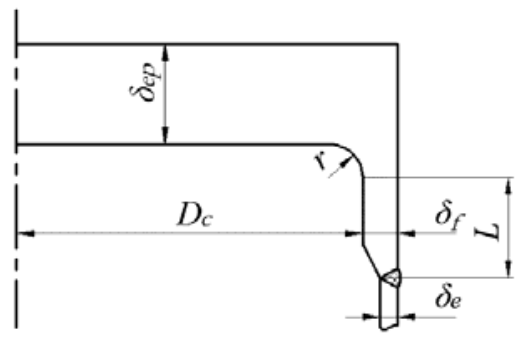

Fig.4 Model parameter of flat cover cylinder structure

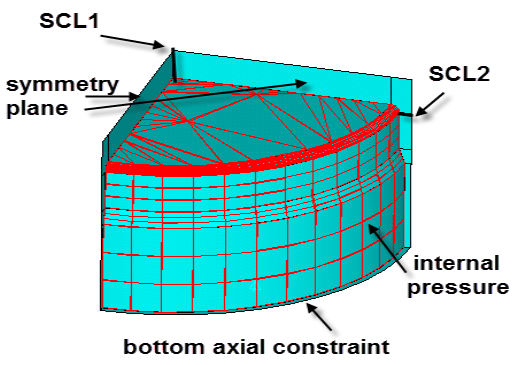

Fig.5 Loading of flat cover cylinder structure

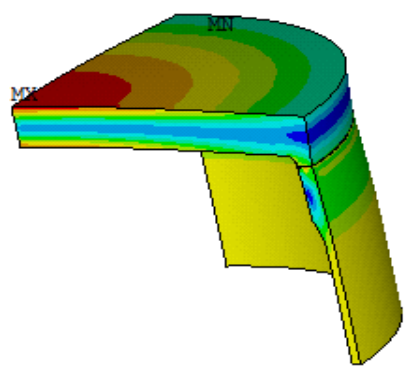

Fig.6 Primary structure stress contour of flat cover cylinder structure

Create the original structure and rotational-constraint-removed-only primary structure (Fig.1.c type) according to the geometrical dimensions in Table 1. Use ANSYS to build $1 / 4$ of flat cover cylinder structure shown in Figure 4. Element solid 185 is used to mesh the model. Stress classification line SCL1 is at the center of the flat head and perpendicular to its wall. Stress classification line SCL2 is at the chamfer of the joint and perpendicular to cylinder wall, as shown in Figure 5. After calculation, do the stress linearization to obtain bending stress at the flat head center, and membrane-plus-bending stress at the joint. For primary structure, only do the stress linearization along stress classification line SCL1 to obtain bending stress at the center of flat head. The results are shown in Table 1, and all the stress intensities are calculated based on the third strength theory. 
Table 1 Comparison of numerical result in original structure and primary structure of the flat cover cylinder structure

\begin{tabular}{|c|c|c|c|c|c|c|c|c|c|}
\hline \multirow{3}{*}{$\begin{array}{c}\text { Calculated } \\
\text { thickness } \\
\text { of flat head } \\
\delta \mathrm{p} / \mathrm{mm}\end{array}$} & \multirow{2}{*}{$\begin{array}{l}\text { Radius } \\
\text { of } \\
\text { rounding }\end{array}$} & \multirow{3}{*}{$\begin{array}{c}\text { calculated } \\
\text { pressure } \\
\mathrm{Pc} / \mathrm{MPa}\end{array}$} & \multirow{3}{*}{$\begin{array}{c}\text { Inner } \\
\text { diameter } \\
\mathrm{Di} / \mathrm{mm}\end{array}$} & \multirow{3}{*}{$\begin{array}{c}\text { Calculated } \\
\text { thickness } \\
\text { of cylinder } \\
\text { shell } \\
\delta / \mathrm{mm}\end{array}$} & \multirow{3}{*}{$\begin{array}{c}\text { Effective } \\
\text { thickness } \\
\text { of } \\
\text { cylinder } \\
\text { shell } \\
\delta \mathrm{e} / \mathrm{mm}\end{array}$} & \multirow{3}{*}{ K } & \multicolumn{2}{|c|}{ Original structure } & \multirow{2}{*}{\begin{tabular}{|c|}
$\begin{array}{c}\text { Primary } \\
\text { structure }\end{array}$ \\
$\begin{array}{l}\text { Center of } \\
\text { flat head }\end{array}$ \\
\end{tabular}} \\
\hline & & & & & & & Center of & Edge of & \\
\hline & $\mathrm{r} / \mathrm{mm}$ & & & & & & $\begin{array}{c}\text { flat head } \\
\mathrm{PL}+\mathrm{Pb} / \mathrm{MPa}\end{array}$ & PL $+\mathrm{Pb}$ & $\begin{array}{c}\text { flat head } \\
\mathrm{PL}+\mathrm{Pb} / \mathrm{MPa}\end{array}$ \\
\hline 16 & 41 & 5 & 2 & 27 & 60 & 0.189 & & & 259.8 \\
\hline 18 & & 5 & 24 & & 60 & 0.1 & & & \\
\hline 199 & & 5 & 2600 & $x$ & 60 & 0.199 & & & \\
\hline 216 & 54 & 5 & 2800 & 41 & 60 & 0.202 & 206.4 & 404.1 & 244.2 \\
\hline
\end{tabular}

\subsection{Calculation analysis}

The results in Table 1 are discussed as follows.

(1) In all original structures of each example, membrane-plus-bending stress at the joint of the flat head-cylinder shell structure is greater than the allowable value of SIII. They can be classified as secondary stress or not, that depends on whether "they are required to maintain the bending stress in the center region within acceptable limits". So remove the rotational constraint at the joint of the flat head-cylinder shell structure to construct a primary structure.

(2) In the primary structure, the bending stress at the cylinder edge disappears, and the local membrane stress of each example is smaller than the allowable value of $\mathrm{S}_{\text {II. }}$ And the maximum bending stress at the center of the flat cover in the primary structure is $259.8 \mathrm{MPa}$, which is smaller than $261 \mathrm{MPa}$. So this primary structure is a kind of structure that can bear loads, and its stresses can meet the requirements of the primary stress assessment. It shows that the disappeared bending stress can be classified as secondary stress. But the stress of flat cover in the primary structure is bigger than that in the original structure by comparison. It is indicated that the rotation constraint removed is advantageous constraint for flat cover. The rotation constraint can't be removed if a thinner wall is designed.

(3) Assess the membrane-plus-bending stress at the joint of the flat head-cylinder shell structure in original structure as secondary stress, its maximum value is $404.1 \mathrm{MPa}$, and is far less than the allowable value of $\mathrm{S}_{\text {IV }} 522 \mathrm{MPa}$. Therefore the structure is safe.

(4) After removing constraints, stresses of the primary structure will redistribute with respect to the original structure. The stress redistribution is beneficial to the part that eliminates high stress (cylinder shell in this example), while adversely to the other part (flat head in this example), in which the primary stress will increase. As for this example, the maximum stress of the flat head is $206.4 \mathrm{MPa}$ in the original structure, and there is still a large margin from the allowable value $261 \mathrm{MPa}$. So it can sequentially bear the load that transferred from the removed constraints. If the safety margin of flat head is not large enough, after removing the constraints, the bending stress at the center of flat head will exceed the allowable value. And it is unable to construct a primary structure by this way. The solution to reduce the stress value at the edge of the cylinder is increasing the wall thickness of the cylinder, taking the original structure as the primary structure. So that the maximum stress value is less than SIII.

\section{References}

[1] Lu Mingwan. Analysis of the general design guidelines, Training course data of China National Standardization Committee on Pressure Vessels. Canton, 1988, 1. (in Chinese)

[2] JB4732-1995, Steel Pressure Vessels-Desgin by Analysis (2005 confirmed) [S]. Beijing: China Machine Press, 2005.

[3] Ming-Wan Lu, LI Jian-Guo. Primary Structure - An Important Concept to Distinguish Primary Stresses J. Seismic Engineering, 340 (1996) 357-363.

[4] Ming-Wan Lu, Yong Chen, Jian-Guo LI. Two-Step Approach of Stress Classification and Primary Structure Method J. Pressure Vessel Technology, 122 (2000) 2-8. 
[5] Wen-Huan Guan.The Implementation of Primary Structure Method in Design by Analysis J.Chemical Machinery, 39 (2012) 187-189. (in Chinese) 